Esta obra forma parte del acervo de la Biblioteca Jurídica Virtual del Instituto de Investigaciones Jurídicas de la UNAM

\title{
EL FENÓMENO DE LAS CANDIDATURAS INDEPENDIENTES EN MÉXICO. ANÁLISIS DE SU IMPLEMENTACIÓN Y PRIMEROS RESULTADOS EN EL PROCESO ELECTORAL 2015
}

\author{
Carlos BÁEz SILVA ${ }^{1}$ \\ Martha Alejandra Tello MendozA²
}

\section{Sumario:}

I. Antecedentes

II. Un modelo nacional de candidaturas independientes

III. Tratamiento de las normas legales y reglamentarias para el registro y desarrollo de la campaña electoral, entre los partidos políticos y los candidatos independientes

IV. Acceso a radio y televisión

V. El acceso al financiamiento público para realizar campañas electorales

VI. El requisito de obtener apoyo ciudadano para poder ser candidato independiente

VII. Comportamiento de las candidaturas independientes como fenómeno luego de la reforma electoral 2014

VIII. Efectividad de las candidaturas independientes frente a los objetivos de la reforma

IX. Fuentes consultadas

\section{ANTECEDENTES}

La transición democrática en México, como en otros países, ${ }^{3}$ tuvo como eje principal la

\footnotetext{
${ }^{1}$ Director del Centro de Capacitación Judicial Electoral del TEPJF. Investigador nacional nivel I.

${ }^{2}$ Asesora en la Unidad General de Transparencia y Sistematización de la Información Judicial de la SCJN.

${ }^{3}$ Para analizar las experiencias de candidaturas independientes en otros países véase, Astudillo, César, "La postulación de candidaturas partidistas e independientes en América Latina en el contexto de los derechos
} 
Esta obra forma parte del acervo de la Biblioteca Jurídica Virtual del Instituto de Investigaciones Jurídicas de la UNAM www.juridicas.unam.mx

construcción de una forma de acceso al poder representativa y plural, lo cual se pensó sólo podía conseguirse mediante la construcción de un sistema de partidos. No es casual entonces que la Ley Electoral Federal de 1946 estableciera en su artículo 60 que "solamente los partidos podrán registrar candidatos". ${ }^{4}$

En 2005, Jorge G. Castañeda solicitó al Instituto Federal Electoral su registro como candidato a la presidencia de la República. Ante la negativa del Instituto, Castañeda recurrió la decisión. La Suprema Corte de Justicia de la Nación (SCJN), al resolver el amparo en revisión 743/2005 decidió sobreseerlo, argumentado la improcedencia del mismo en materia electoral pero, a partir de la ruta emprendida por el ex canciller, el tema de las candidaturas independientes se puso de relieve nuevamente. El resultado final fue una modificación normativa controvertida que tuvo como prolegómeno una sentencia condenatoria para el Estado mexicano por parte de la Corte Interamericana de Derechos Humanos por carecer de un recurso efectivo tratándose del control de constitucionalidad de leyes electorales.

En 2007 la pinza se cerró al establecerse en la Constitución Política de los Estados Unidos Mexicanos que el derecho a registrar candidatos - tanto a nivel local como

de participación política", en Freidenberg, Flavia y Muñoz Pogossian, Betilde (eds.), Las reformas a las organizaciones de partidos en América Latina, Lima, Pontificia Universidad Católica, Organización de los Estados Americanos, UNAM, Instituto de Investigaciones Jurídicas, Sociedad Argentina de Análisis Político, 2015; Bhattacharya, Kaushik, "Independent Candidates in a Parliamentary Election in India: A Poisson Regression Model", Working Paper Series: 2009/10-034, Prabandh Nagar, Indian Institute of Management Lucknow; Bolleyer, Nicole y Weeks, Liam, "The Puzzle of Non-Party Actors in Party Democracy: Independents in Ireland", Comparative European Politics, vol. 7 (3), 2007, pp. 299-324; Brancati, Dawn, “Winning Alone: The Electoral Fate of Independent Candidates Worldwide", Journal of Politics, vol. 70 (3), 2008, pp.648-662; Došek, Tomáš, "La presencia y el éxito de los independientes en las elecciones subnacionales en Chile (2004-2012)", XI Congreso Chileno de Ciencia Política (ACCP), Política, diversidad y conflicto, nuevos debates a 30 años de la ACCP, Santiago de Chile, 15 al 17 de octubre de 2014; Hale, Henry, Why not Parties in Russia?, Cambridge, Cambridge University Press, 2007; Ishiyama, John, Batta, Anna et al.,"Political Parties, Independents and the Electoral Market in Sub-Saharan Africa", Party Politics, vol. 19 (5), 2011, pp.695-712, Moser, Robert, "Independents and Party Formation: Elite Partisanship as an Intervening Variable in Russian Politics", Comparative Politics, vol. 31 (2), 1999, pp. 147-165; Rodrigues, Mark y Brenton, Scott, "The Age of Independence? Independents in Australian Parliaments", Australasian Parliamentary Review, vol. 25 (1), 2010, pp. 109-135; Weeks, Liam, “We don't like (to) party. A typology of Independents in Irish Political Life, 19222007", Irish Political Studies, vol. 24 (1), 2009, pp. 1-27.

${ }^{4}$ García Orozco, 1987, p.247. 
Esta obra forma parte del acervo de la Biblioteca Jurídica Virtual del Instituto de Investigaciones Jurídicas de la UNAM www.juridicas.unam.mx

federal - era exclusivo de los partidos políticos, quedando cerrada la posibilidad a la existencia de candidaturas independientes.

Sin embargo, el poder reformador de la Constitución decidió desandar el camino seguido hasta entonces, a través de decretos publicados en el Diario Oficial de la Federación del 9 de agosto de 2012 y 27 de diciembre de 2013, en los cuales se reconoció el derecho de los ciudadanos a postularse a cargos de elección popular de manera independiente de los partidos políticos.

No obstante, las candidaturas independientes no son nuevas en el sistema jurídico mexicano, pues ya habían sido incluidas en las leyes electorales federales de 1911, 1916, y $1918 .{ }^{5}$ Asimismo, en el constitucionalismo local mexicano y en la actividad jurisdiccional, atravesaron por diversos episodios. Aún y cuando las candidaturas independientes desaparecieron a nivel federal, en el ámbito local no existió prohibición expresa, sino hasta la reforma constitucional del 13 de noviembre de 2007 que modificó el artículo 116.

En Tamaulipas, en 1998, una ciudadana que participó en las elecciones como candidata sin registro ganó la elección municipal del ayuntamiento de Jiménez. La autoridad administrativa electoral en un primer momento se rehusó a entregar la constancia de mayoría, no obstante, el tribunal electoral de la entidad confirmó su victoria al estimar que constituía una opción que permitía al ciudadano que no simpatizaba con ninguna de las corrientes políticas registradas, a "proponer una persona de su satisfacción, siendo esto una vía alterna para llegar a ser parte integrante de los poderes públicos, en el caso de obtener el voto de las mayorías". ${ }^{6}$

En Michoacán, en 2001 (SUP-JDC-037-2001), un ciudadano solicitó su registro como candidato independiente a la gubernatura. Ante la negativa del instituto local, el aspirante acudió al Tribunal Electoral del Poder Judicial de la Federación (TEPJF), el cual estimó que si bien no existía disposición expresa en la Constitución respecto del derecho de la ciudadanía a aspirar a una candidatura independiente, el derecho a ser votado era fundamental, y al no existir restricción expresa sobre el tema (dado que la exclusividad

\footnotetext{
${ }^{5}$ Becerra, Pablo, "Las candidaturas independientes en México. Una vía para ampliar la participación ciudadana", en Gilas, Karolina y Medina Torres, Eduardo (eds.), Candidaturas independientes: desafíos y propuestas, México, TEPJF, Instituto Electoral de Morelos, Tirant lo Blanch, 2014, pp. 99-229.

${ }^{6}$ Campos, 2006, p.246.
} 
Esta obra forma parte del acervo de la Biblioteca Jurídica Virtual del Instituto de Investigaciones Jurídicas de la UNAM

en el registro para los partidos era sólo respecto de diputados y senadores federales electos vía representación proporcional) era viable la postulación por este medio.

En Veracruz, en el 2001, la Sala Superior estableció un criterio importante. Un candidato no registrado intentó formar parte de un ayuntamiento. Si bien se consideró que eventualmente, según la legislación estatal, un candidato no registrado no podría obtener la constancia de mayoría - de ser el caso - los votos emitidos en su favor no debían considerarse nulos ni válidos, sino que tendrían que computarse por separado en una tercera categoría específica. Lo anterior consta en la sentencia SUP-JDC-713/2004.

A nivel local, la Constitución del Estado de Yucatán —vigente hasta 2009- contempló la existencia de candidaturas independientes y la figura estuvo regulada en la Ley de Instituciones y Procedimientos Electorales de dicha entidad. La Sala Superior del Tribunal Electoral del Poder Judicial de la Federación llegó, incluso, a declarar la validez de una elección de ayuntamiento en la cual el ganador era un candidato apartidista (SUP-JRC86/2007).

En Sonora, la ley electoral local contenía el título III, capítulo VI, dedicado —en su totalidad - a la reglamentación detallada de la figura de las candidaturas independientes, pero fue abrogada en 2008.

De lo anterior se observan importantes antecedentes, los cuales sin duda impactaron en las discusiones que desembocaron finalmente en la apertura del sistema de partidos en todo el país. De esta forma, en la exposición de motivos se plasmó que "las candidaturas Independientes son una vía de participación de los ciudadanos en los asuntos públicos en un régimen democrático. La apertura en esta materia, significa un avance en la construcción de nuestra democracia". Por lo cual a partir de este momento, se abrió una nueva vía de acceso a la representación pública.

\section{UN MODELO NACIONAL DE CANDIDATURAS INDEPENDIENTES}

El 23 de mayo de 2014 se publicó en el Diario Oficial de la Federación, la Ley General de Instituciones y Procedimientos Electorales, en adelante LGIPE. La normatividad relativa a las candidaturas independientes quedó estipulada en el libro séptimo de los artículos 357-439.

De un análisis de esta ley, así como de la legislación de aquellas entidades federativas en que la materia ha sido legislada, se observa que en términos generales se siguió el 
Esta obra forma parte del acervo de la Biblioteca Jurídica Virtual del Instituto de Investigaciones Jurídicas de la UNAM www.juridicas.unam.mx

mismo esquema de regulación. Básicamente, las normas federales, contenidas en una ley general, fueron el modelo a seguir en los congresos estatales. Por esta razón, se habla con fines meramente expositivos de un modelo nacional de candidaturas independientes, con ciertas particularidades en las entidades federativas.

Con algunas variaciones, en todos los estados se requiere: 1) que se emita la convocatoria correspondiente; 2) que los ciudadanos interesados en registrarse, presenten su manifestación de intención por escrito ante los órganos de las autoridades electorales correspondientes; 3) para ello deben, entre otras cuestiones, constituir una asociación civil y nombrar a un responsable de la administración de los recursos financieros, y 4) el esquema de financiamiento es el siguiente: privado para la etapa de la obtención del apoyo ciudadano, y público y privado para la etapa de las campañas.

Con diferencias en los porcentajes y el número de ciudadanos que pueden obtener el registro por tipo de elección, en todas las entidades se requiere de un número específico de firmas de apoyo ciudadano. Los aspirantes que obtengan la calidad de candidato independiente tienen acceso básicamente a las mismas prerrogativas en todos los estados, entre las más importantes se encuentran el acceso a tiempo en radio y televisión, y financiamiento público. Finalmente, todas, al ser el Instituto Nacional Electoral (INE) el responsable de fiscalizar tanto las elecciones federales como las locales, siguen el mismo tipo regulación en materia de fiscalización.

El siguiente esquema resume el proceso básico de este modelo nacional de candidaturas independientes: ${ }^{7}$

Figura 1

Modelo nacional de candidaturas independientes

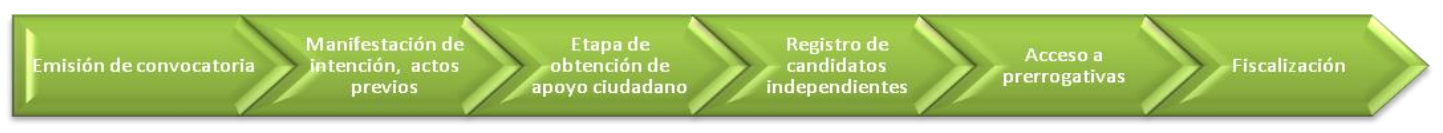

Fuente: elaboración propia a partir de la legislación aplicable, federal y local, vigente

\footnotetext{
${ }^{7}$ Sobre el tema específico de la regulación de las candidaturas independientes, véase Valdez, 2014; Agíss, 2014; Gilas, 2015; Arellano, 2015.
} 
Esta obra forma parte del acervo de la Biblioteca Jurídica Virtual del Instituto de Investigaciones Jurídicas de la UNAM

durante el proceso electoral de 2015.

\section{TRATAMIENTO DE LAS NORMAS LEGALES Y REGLAMENTARIAS PARA EL REGISTRO Y DESARROLLO DE LA CAMPAÑA ELECTORAL, ENTRE LOS PARTIDOS POLÍTICOS Y LOS CANDIDATOS INDEPENDIENTES}

Se ha criticado en diferentes medios de comunicación y distintos foros, el tratamiento legal y reglamentario que se da a los partidos políticos y a los candidatos independientes. Lo anterior, en cuanto al registro y desarrollo de las campañas políticas, fundamentalmente por la diferencia que existe entre ellos. Se argumenta que se da un tratamiento igual a quienes no lo son, ya que mientras el partido cuenta con diversos recursos materiales y humanos, el independiente no cuenta con ellos y realiza los actos de precampaña y campaña con recursos menores y con la asistencia de personal muy reducido, lo cual implica una desigualdad en la contienda y puede resultar una diferencia importante en los resultados de la votación.

Mientras los partidos políticos tienen un proyecto de permanencia en el tiempo, que tiene por objeto allegarse de simpatizantes y militantes que auxiliarán al partido con su voto en distintas elecciones para formar tal gobierno, los independientes tienen un proyecto que se extingue naturalmente con la elección, y su pretensión se circunscribe al resultado de la contienda correspondiente y no pretende la formación de cuadros o de estructuras de gobierno permanentes, por lo cual resulta incorrecto asemejar a dos estructuras que son esencialmente distintas. Mientras los partidos políticos viven y realizan sus actividades permanentes con base en los recursos que les son proporcionados por el Estado en forma permanente y de acuerdo con los resultados que mantenga en la actividad política, los candidatos independientes no cuentan con esos recursos en forma permanente sino sólo durante el proceso electoral respectivo, que limita la posibilidad de construir economías de escala que sirvan para todas las actividades políticas que desarrollen, incluyendo las distintas campañas políticas que emprendan.

El apoyo ciudadano que deben reunir los candidatos independientes como requisito para la obtención de su registro como aspirantes, es excesivo y desproporcionado, toda vez que se les exige un número muy alto de firmas para la obtención del registro —el 
Esta obra forma parte del acervo de la Biblioteca Jurídica Virtual del Instituto de Investigaciones Jurídicas de la UNAM www.juridicas.unam.mx

DOI: http://dx.doi.org/10.22201/iij_.24487910e.2015.7.10091

estándar internacional es del $1 \%-{ }^{8}$ y se establecen mecanismos que resultan insostenibles, tales como la presentación de la credencial para votar y el llenado de un formulario que atenta contra la privacidad de la preferencia electoral, aun cuando éste no puede entenderse como la expresión de un voto como tal.

Se requiere mayor sensibilización en el sentido de la independencia de dicho formato con la expresión definitiva de un voto en casilla, es decir, la expresión de una simpatía del derecho a participar en la jornada electoral por un candidato independiente no significa una expresión de voto en la elección respectiva, ni el compromiso o promesa de voto, sino una expresión de simpatía respecto a la participación de ese candidato independiente en el proceso electoral, con independencia del resultado obtenido en las urnas. En efecto, los ciudadanos en general no tienen la visión de esta circunstancia y pueden confundir que la información expresada en ese documento y la inclusión de su credencial electoral, podría difundirse en su perjuicio entre las autoridades del país.

\section{ACCESO A RADIO Y TELEVISIÓN}

Los candidatos independientes pueden tener acceso a radio y televisión en los tiempos que son reservados al Estado y administrados por el INE, en los términos establecidos por el artículo 41, fracción III, de la Constitución. Este acceso se refiere a las prerrogativas para las campañas electorales y la transmisión de la propaganda política e imágenes de los candidatos independientes, de la misma forma en que lo hacen los partidos políticos, siempre que sean contratados directamente por el INE, siguiendo al efecto los lineamientos autorizados por las leyes de la materia.

Para lo anterior, los tiempos se distribuyen de la siguiente manera, el 30\% de forma igualitaria entre los candidatos y partidos en su conjunto. Como lo establece la LGIPE en su artículo 173, son los OPLES quienes están facultados para señalar los tiempos que a cada uno corresponden en relación a las jornadas electorales coincidentes, destinando quince minutos diarios en cada estación y canal repartido entre los partidos y los candidatos independientes.

Siendo el Comité de Radio y Televisión (CRT) la entidad responsable de conocer y

\footnotetext{
${ }^{8}$ La Comisión de Venecia emitió durante su 51 a reunión plenaria del 5 y 6 de julio de 2002, el Código de buenas prácticas en materia electoral. Respecto a la presentación de candidaturas, en la directriz 1.3 estableció que "con el fin de evitar manipulaciones de ese tipo, es preferible que la ley no exija las firmas de más del $1 \%$ de los votantes".
} 
Esta obra forma parte del acervo de la Biblioteca Jurídica Virtual del Instituto de Investigaciones Jurídicas de la UNAM

aprobar las pautas de transmisión (LGIPE, artículo 184), y ante las variaciones temporales que pudieran surgir entre las jornadas comiciales del Proceso Electoral 2014-2015, este órgano determinó aprobar diversos modelos de pautas atendiendo a las particularidades de cada entidad federativa antes de que las candidaturas fueran aprobadas (INE/ACRT/17/2015), previniendo así los tiempos en medios de comunicación en atención al número de candidatos, bajo diferentes supuestos contemplados en los acuerdos del INE. Sin embargo, cabe destacar que la sustitución de pautas no fue una figura contemplada en todas las entidades, puesto que la misma sólo se aplicó en el $25 \%$ de los casos (de un total de 16 entidades), mientras que el resto no hizo modificaciones ante la falta de celeridad del proceso de procedencia de registros de candidaturas independientes en Campeche, Distrito Federal, Guerrero, Estado de México, Michoacán, Morelos, Sonora y Tabasco (IRC, p. 20).

En este punto, conviene señalar que dichas modificaciones responden al modelo instaurado en la legislación, en el cual, el o los candidatos independientes involucrados en una elección accederán a los tiempos destinados para su propaganda bajo la figura de un partido de nuevo registro (LGIPE, artículo 412).

\section{EL ACCESO AL FINANCIAMIENTO PÚBLICO PARA REALIZAR CAMPAÑAS ELECTORALES}

El acceso al financiamiento es fundamental para los candidatos, toda vez que las elecciones y el contexto general electoral requiere de un monto importante de recursos económicos para hacer posible el proselitismo y dar a conocer la ideología de los candidatos. La obtención de recursos y la realización de actos de campaña es esencial para el ejercicio del derecho a ser votado, por ello, no se puede reconocer de forma plena el derecho a ser votado por la vía independiente sino es posible obtener recursos con la finalidad de hacer campaña (SM-JDC-429/2015, 11).

Lo anterior se relaciona directamente con el ejercicio a la libertad de expresión del candidato así como con el derecho a la información por parte del electorado. El hecho de transmitir las ideas a la ciudadanía es una actividad demandante tanto en recursos económicos como humanos. De esta forma, el financiamiento tiene tal importancia que no hay duda de su influencia directa en los resultados de los comicios; por ello, el Estado establece reglas estrictas para el acceso y los límites del financiamiento así como los procesos de fiscalización para garantizar la autenticidad y transparencia de los comicios. 
Esta obra forma parte del acervo de la Biblioteca Jurídica Virtual del Instituto de Investigaciones Jurídicas de la UNAM www.juridicas.unam.mx

La LGIPE contiene las reglas aplicables a los candidatos independientes. En primer lugar, la ley señala que el régimen de financiamiento es de carácter mixto; es decir, los candidatos pueden usar recursos de origen público y privado. En cuanto al financiamiento privado, éste sólo puede conformarse mediante las aportaciones del candidato independiente y simpatizantes, pero no puede ser superior al $10 \%$ del monto señalado como tope de gastos de campaña para la elección que se trate.

Con base en la libre configuración legislativa, las legislaciones locales establecieron diferentes topes de financiamiento privado, de tal forma que se pueden clasificar en:

a) Entidades que siguieron el modelo federal.

b) Entidades que establecieron un límite diferente al modelo Federal.

c) Entidades que fueron omisas.

Ahora bien, los candidatos independientes deben: (i) realizar todas las operaciones en relación con los recursos de la campaña electoral mediante la cuenta bancaria que se abrió desde la etapa de registro (LGIPE, artículo 403); (ii) recibir todas las aportaciones mediante cheque o transferencia bancaria (LGIPE, artículo 403); (iii) pagar los gastos mediante cheque nominativo o transferencia electrónica y conservar la documentación probatoria ${ }^{9}$ que exige la ley (LGIPE, artículo 404), y (iv) destinar las aportaciones únicamente para los fines de la candidatura independiente (LGIPE, artículo 405).

En cuanto al financiamiento público, los candidatos independientes tienen derecho a recibirlo, pero la ley considera al conjunto de candidatos como un partido político de recién registro; es decir, todos los candidatos independientes que participen para los

\footnotetext{
${ }^{9}$ Artículo 404:
}

1. Todo egreso deberá cubrirse con cheque nominativo o transferencia electrónica. En el caso de los pagos por la prestación de bienes o servicios, adicionalmente el cheque deberá contener la leyenda "para abono en cuenta del beneficiario". Las pólizas de los cheques deberán conservarse anexas a la documentación comprobatoria junto con la copia del cheque a que se hace referencia.

2. Los comprobantes que amparen los egresos que realicen los Candidatos Independientes, deberán ser expedidos a su nombre y constar en original como soporte a los informes financieros de las campañas electorales, los cuales estarán a disposición de la unidad de fiscalización del Instituto para su revisión de conformidad con lo dispuesto en esta Ley. Dicha documentación deberá cumplir con los requisitos que exigen las disposiciones fiscales aplicables, así como las establecidas por el Reglamento de Fiscalización de la Unidad referida. 
Esta obra forma parte del acervo de la Biblioteca Jurídica Virtual del Instituto de Investigaciones Jurídicas de la UNAM www.juridicas.unam.mx

DOl: http://dx.doi.org/10.22201/iij.24487910e_2015.7.10091

distintos cargos de elección popular pueden acceder a la parte igualitaria del financiamiento público como si fueran un partido político.

A pesar de que en el proceso electoral ordinario federal (2014-2015) únicamente se renovaron las diputaciones, este esquema de repartición de financiamiento público resulta relevante, toda vez que la mayoría de las entidades federativas lo replicaron para la renovación de los cargos de gobernador-jefe de Gobierno, diputados locales y ayuntamientos-delegaciones. Por ello, todos los estados consideraron al conjunto de candidatos independientes como un partido político de nueva creación.

En un principio, los debates relacionados con la equidad en la contienda se centraron en el régimen de distribución del financiamiento público, toda vez que era claro que los candidatos independientes nunca obtendrían el mismo monto de financiamiento como el que lograría un partido político de reciente registro. Así, esta preocupación se manifestó ante la SCJN mediante la acción de inconstitucionalidad 22/2014 y sus acumuladas 26/2014, 28/2014 y 30/2014.

Por otra parte, otro de los temas discutidos versó sobre el hecho de que los candidatos independientes no pueden recibir aportaciones de dinero en efectivo como lo hacen los partidos políticos. De forma muy similar, la Corte argumentó que los candidatos independientes no tienen una permanencia en elecciones subsecuentes como los partidos políticos, por lo que la fiscalización periódica no es posible. Finalmente, la Corte señaló que los candidatos independientes deben evitar a toda costa el uso de recursos cuya identificación sea compleja para efectos del proceso de fiscalización.

\section{EL REQUISITO DE OBTENER APOYO CIUDADANO PARA PODER SER CANDIDATO INDEPENDIENTE}

Los aspirantes a candidatos independientes deberán obtener, entre otros requisitos, la expresión de apoyo ciudadano. Los aspirantes son los únicos que tienen el derecho de conseguir firmas y los datos necesarios para integrar las cédulas de apoyo ciudadano. Para ello, los aspirantes deben hacerlo conforme a los plazos y porcentajes que señala la ley para cada cargo en concreto; asimismo, el aspirante debe abstenerse en todo momento de realizar actos anticipados de campaña, propaganda negativa, y contratar publicidad en radio y televisión (LGIPE, artículos 320 y 380, incisos e) y f)).

Es importante mencionar que a partir de que se obtiene la calidad de aspirante a 
Esta obra forma parte del acervo de la Biblioteca Jurídica Virtual del Instituto de Investigaciones Jurídicas de la UNAM www.juridicas.unam.mx

candidato independiente también se adquieren los derechos que señala la ley, cuyo ejercicio está relacionado directamente con la obtención del apoyo ciudadano. De acuerdo al artículo 379 de la LGIPE, algunos de estos derechos son:

I. Realizar actos para promover sus ideas y propuestas;

II. Usar financiamiento privado;

III. Nombrar un representante para asistir a las sesiones del Consejo General, pero sin derecho a voz ni voto;

IV. Insertar la leyenda "Aspirante a Candidato Independiente" en la propaganda

Como consecuencia de los esfuerzos del Estado por mantener una contienda equitativa entre todos los candidatos, ya sean independientes o propuestos por partidos políticos, la LGIPE establece disposiciones en materia de financiamiento y fiscalización, aplicables a las operaciones relacionadas con los actos para la obtención del apoyo ciudadano. Por lo tanto, el aspirante debe:

I. Usar en todo momento la cuenta que abrió a nombre de la Asociación Civil;

II. Financiar los actos con recursos privados de procedencia lícita;

III. No recibir aportaciones en efectivo, metales ni piedras preciosas;

IV. Respetar los topes de gastos que determine el Consejo General ( $10 \%$ del establecido para las campañas inmediatamente anteriores);

V. Pagar los gastos mediante cheque nominativo o transferencia bancaria;

VI. Tener comprobantes originales de los gastos expedidos a nombre del aspirante y de la persona encargada del manejo de los recursos financieros (firmas mancomunadas);

VII. Nombrar a una persona encargada de los recursos y la presentación de informes; y

VIII. Entregar el informe de ingresos y egresos dentro de los 30 días siguientes a la conclusión del periodo para recabar el apoyo ciudadano. (LGIPE, artículos 373-378 y 380 , inciso c)).

Asimismo, la legislación prohíbe el apoyo propagandístico o económico por parte de extranjeros; ministros de culto y asociaciones religiosas; poderes de la Federación; entidades federativas y ayuntamientos; dependencias, entidades y organismos 
Esta obra forma parte del acervo de la Biblioteca Jurídica Virtual del Instituto de Investigaciones Jurídicas de la UNAM www.juridicas.unam.mx

DOI: http://dx.doi.org/10.22201/iij_.24487910e_2015.7.10091

autónomos de cualquier nivel; partidos políticos nacionales y organismos internacionales; personas morales y personas que vivan o trabajen en el extranjero (LGIPE, artículo 380, inciso d).

Por otro lado, las obligaciones de los partidos políticos son menos estrictas que las impuestas a los independientes, pues los partidos y sus candidatos cuentan en todo momento con la estructura orgánica y financiera del propio partido para desarrollar todas las funciones y actividades que les son propias.

\section{COMPORTAMIENTO DE LAS CANDIDATURAS INDEPENDIENTES COMO FENÓMENO LUEGO DE LA REFORMA ELECTORAL 2014}

El 23 de noviembre, el INE publicó la convocatoria para todos aquellos ciudadanos interesados en participar como candidatos independientes a diputados federales en el proceso electoral 2014-2015. En un primer momento, 122 ciudadanos (10 mujeres y 112 hombres) presentaron su manifestación de intención. No obstante, sólo 57 aspirantes (46\%) lo consiguieron (7 mujeres y 50 hombres). Del 30 de diciembre de 2014 al 27 de febrero de 2015, estos 57 aspirantes buscaron el apoyo de la ciudadanía, sin embargo, sólo 22 lograron cubrir todos los requisitos que representa el $42 \%$ del total de aspirantes (INE, 2015).

De acuerdo al cómputo distrital del INE, estos fueron los resultados obtenidos:

Cuadro 2

Cómputos distritales candidatos independientes federales

\begin{tabular}{|c|c|c|c|c|c|}
\hline Núm & Candidato independiente & Entidad & Distrito & $\begin{array}{l}\text { Votació } \\
n\end{array}$ & Porcentaje \\
\hline 1 & Rivera Figueroa Sergio & Chihuahua & III, Juárez & 1,749 & $2.28 \%$ \\
\hline 2 & Rosales Ávalos Eliseo & $\begin{array}{l}\text { Distrito } \\
\text { Federal }\end{array}$ & XXIV, Coyoacán & 6,471 & $5.02 \%$ \\
\hline 3 & Mota Rojas Antonio & Hidalgo & $\begin{array}{l}\text { VI, Pachuca de } \\
\text { Soto }\end{array}$ & 23,862 & $18.05 \%$ \\
\hline 4 & Ibarra Ocampo Carlos Manue & I) Nayarit & II, Tepic & 9,008 & $7.49 \%$ \\
\hline 5 & Sandoval De León Blanca Lilia & Nuevo León & VIII, Guadalupe & 15,421 & $9.87 \%$ \\
\hline
\end{tabular}


Esta obra forma parte del acervo de la Biblioteca Jurídica Virtual del Instituto de Investigaciones Jurídicas de la UNAM www.juridicas.unam. $\mathrm{mx}$ https://biblio.juridicas.unam.mx/bjv https://revistas.juridicas.unam. $\mathrm{mx} /$

DOI: http://dx.doi.org/10.22201/iij_.24487910e_2015.7.10091

\begin{tabular}{|c|c|c|c|c|c|}
\hline Núm & Candidato independiente & Entidad & Distrito & $\begin{array}{l}\text { Votació } \\
n\end{array}$ & Porcentaje \\
\hline 6 & $\begin{array}{l}\text { Merlo Martínez Manuel } \\
\text { Alberto }\end{array}$ & Puebla & VI, Puebla & 5,208 & $5.13 \%$ \\
\hline 7 & $\begin{array}{l}\text { Hernández Barbosa Jesús } \\
\text { Amador }\end{array}$ & Puebla & XV, Tehuacán & 13,990 & $12.89 \%$ \\
\hline 8 & $\begin{array}{l}\text { Ruiz Morcillo } \\
\text { Florentino }\end{array}$ & $\begin{array}{l}\text { Quintana } \\
\text { Roo }\end{array}$ & $\begin{array}{l}\text { II, Othón P. } \\
\text { Blanco }\end{array}$ & 10,969 & $8.12 \%$ \\
\hline 9 & Ayala López Jesús Alfredo & Sinaloa & I, El Fuerte & 19,753 & $19.65 \%$ \\
\hline 10 & $\begin{array}{l}\text { Clouthier Carrillo Manuel } \\
\text { Jesús }\end{array}$ & Sinaloa & V, Culiacán & 43,730 & $42.45 \%$ \\
\hline 11 & $\begin{array}{l}\text { Corrales Burgueño Víctor } \\
\text { Antonio }\end{array}$ & Sinaloa & VI, Mazatlán & 15,297 & $16.35 \%$ \\
\hline 12 & Jiménez Ramírez José Vidal & Sinaloa & VII, Culiacán & 13,629 & $16.77 \%$ \\
\hline 13 & Camacho Castro Giova & Sinaloa & VIII, Mazatlán & 8,909 & $10.55 \%$ \\
\hline 14 & Pulido Santiago Adolfo & Tabasco & III, Comalcalco & 2,321 & $1.34 \%$ \\
\hline 15 & $\begin{array}{l}\text { Chavira Martínez Diana } \\
\text { Elizabeth }\end{array}$ & Tamaulipas & I, Nuevo Laredo & 3,110 & $2.33 \%$ \\
\hline 16 & $\begin{array}{l}\text { Rentería Campos Jorge } \\
\text { Prisciliano }\end{array}$ & Tamaulipas & IV, Matamoros & 8,136 & $6.36 \%$ \\
\hline 17 & $\begin{array}{l}\text { Santillán Martínez Manuel } \\
\text { Heriberto }\end{array}$ & Tamaulipas & $\begin{array}{ll}\text { VII, } & \text { Ciudad } \\
\text { Madero } & \end{array}$ & 4,983 & $3.45 \%$ \\
\hline 18 & Romero López Daniel & Tlaxcala & III, Zacatelco & 2,488 & $2.24 \%$ \\
\hline 19 & Pérez Sánchez Rafael & Veracruz & X, Xalapa & 9,048 & $7.42 \%$ \\
\hline 20 & $\begin{array}{l}\text { De La Torre Jaramillo Eduardo } \\
\text { Sergio }\end{array}$ & Veracruz & X, Xalapa & 2,449 & $2.00 \%$ \\
\hline 21 & Valles Martínez Esteban & Veracruz & XI, Coatzacoalcos & 3,629 & $3.08 \%$ \\
\hline
\end{tabular}


Esta obra forma parte del acervo de la Biblioteca Jurídica Virtual del Instituto de Investigaciones Jurídicas de la UNAM www.juridicas.unam.mx

DOI: http://dx.doi.org/10.22201/iij.24487910e_2015.7.10091

\begin{tabular}{|l|l|l|l|l|l|}
\hline Núm & Candidato independiente & Entidad & Distrito & \multicolumn{1}{l|}{$\begin{array}{l}\text { Votació } \\
n\end{array}$} & Porcentaje \\
\hline 22 & García Alonso Roberto & Veracruz & 11 , Coatzacoalcos & 1,340 & $1.13 \%$ \\
\hline \hline & Total & & 225,500 & $9.27^{10}$ \\
\hline
\end{tabular}

Fuente: $h$ ttp://portales.te.gob.mx/candidaturas-independientes/node/72

De los 22 candidatos independientes, sólo Manuel Jesús Clouthier Carrillo obtuvo la mayoría de votos con 43, 730 (42.45\%). No se pretende establecer símil alguno pero si es una muestra de la competitividad efectiva de estos candidatos, sin embargo, la votación se ubicó en $9.27 \%$, lo que pone de relieve la competitividad de algunos de éstos.

A nivel local, de las 17 entidades federativas con proceso electoral en 2015, 9 tuvieron elección de gobernador: Baja California Sur, Campeche, Colima, Guerrero, Michoacán, Nuevo León, Querétaro, San Luis Potosí y Sonora. Sólo lograron su registro como candidatos independientes tres ciudadanos en Baja California Sur, Campeche y Nuevo León.

De acuerdo a los cómputos correspondientes, estos fueron los resultados obtenidos:

Cuadro 3

Cómputos estatales candidatos independientes a gobernador

\begin{tabular}{|l|l|l|l||}
\hline Entidad & Candidato independiente & Votación & Porcentaje \\
\hline \begin{tabular}{l|l||} 
Baja California \\
Sur
\end{tabular} & Benjamín de la Rosa Escalante & 4,361 & $1.76 \%$ \\
\hline \hline Campeche & Luis Antonio Che Cu & 2,461 & $0.66 \%$ \\
\hline \hline Nuevo León & Jaime Heliodoro Rodríguez & $1,020,552$ & $48.8 \%$ \\
\hline
\end{tabular}

Fuente: $h t t p: / / p o r t a l e s . t e . g o b . m x / c a n d i d a t u r a s-i n d e p e n d i e n t e s / n o d e / 72$

De los tres candidatos, sólo Jaime Heliodoro Rodríguez Calderón “El Bronco" resultó electo, mientras que la votación recibida en conjunto por los otros dos candidatos no alcanzó el 3\%. El resultado obtenido por Rodríguez debe ser analizado en prospectiva en

\footnotetext{
${ }^{10}$ Media del porcentaje obtenido.
} 
Esta obra forma parte del acervo de la Biblioteca Jurídica Virtual del Instituto de Investigaciones Jurídicas de la UNAM www.juridicas.unam.mx

DOI: http://dx.doi.org/10.22201/iij_.24487910e.2015.7.10091

aras de determinar la articulación del sistema de partidos previamente existentes para poder explicar un resultado que supera 20 veces en cuanto al porcentaje de votación al candidato independiente a gobernador que más se le acercó. La media en la votación recibida fue de $17.07 \%$, dato que debe ser manejado cuidadosamente, ya que evidencia que el resultado de Rodríguez en Nuevo León está, auténticamente, fuera de rango.

Respecto a la elección de diputados locales, en 10 entidades federativas de 17, lograron registro como candidatos independientes 29 ciudadanos. A continuación se presenta la relación de los resultados obtenidos por candidato independiente

Cuadro 4

Cómputos distritales candidatos independientes a diputados locales

\begin{tabular}{|l|l|l|l|l|l|}
\hline Núm. & $\begin{array}{l}\text { Candidato } \\
\text { independiente }\end{array}$ & Entidad & Distrito & Votación & $\%$ \\
\hline 1 & Baldemar Sicairos & $\begin{array}{l}\text { Baja California } \\
\text { Sur }\end{array}$ & Distrito I & 374 & $2.27 \%$ \\
\hline 2 & $\begin{array}{l}\text { Franco Adolfo Castro } \\
\text { Álvarez }\end{array}$ & $\begin{array}{l}\text { Baja California } \\
\text { Sur }\end{array}$ & Distrito III & 369 & $2.99 \%$ \\
\hline 3 & $\begin{array}{l}\text { José Lorenzo Cota } \\
\text { Martínez }\end{array}$ & $\begin{array}{l}\text { Baja California } \\
\text { Sur }\end{array}$ & Distrito III & 484 & $3.92 \%$ \\
\hline 4 & Jorge Martínez Ramírez & $\begin{array}{l}\text { Baja California } \\
\text { Sur }\end{array}$ & Distrito V & 679 & $3.37 \%$ \\
\hline 5 & $\begin{array}{l}\text { Irán Silverio Rodríguez } \\
\text { Martínez }\end{array}$ & $\begin{array}{l}\text { Baja California } \\
\text { Sur }\end{array}$ & Distrito XII & 854 & $7.96 \%$ \\
\hline 7 & Muría de los Ángeles & $\begin{array}{l}\text { Baja California } \\
\text { Sur }\end{array}$ & Distrito XIII & 879 & $9.61 \%$ \\
\hline 8 & Jorge Adrián Ojeda Ruiz & Chiapas & Distrito II & 3,021 & $3.53 \%$ \\
\hline 9 & Judith Barrios Bautista & Distrito Federal & Distrito VII & 3,919 & $4.27 \%$ \\
\hline 10 & Efraín Morales Sánchez & Distrito Federal & Distrito XXI & 2,403 & $2.95 \%$ \\
\hline 11 & $\begin{array}{l}\text { Edgar Adán Montero } \\
\text { Distrito Federal }\end{array}$ & $\begin{array}{l}\text { Distrito } \\
\text { XXII }\end{array}$ & 1,517 & $2.00 \%$ \\
\hline
\end{tabular}


Esta obra forma parte del acervo de la Biblioteca Jurídica Virtual del Instituto de Investigaciones Jurídicas de la UNAM www.juridicas.unam.mx

DOI: http://dx.doi.org/10.22201/iij_.24487910e_2015.7.10091

\begin{tabular}{|c|c|c|c|c|c|}
\hline Núm. & $\begin{array}{l}\text { Candidato } \\
\text { independiente }\end{array}$ & Entidad & Distrito & Votación & $\%$ \\
\hline 12 & $\begin{array}{l}\text { José Pedro Kumamoto } \\
\text { Aguilar }\end{array}$ & Jalisco & Distrito $X$ & 57,215 & $\begin{array}{l}37.52 \\
\%\end{array}$ \\
\hline 13 & $\begin{array}{l}\text { Ulises Daniel Ramos } \\
\text { Ramírez }\end{array}$ & México & $\begin{array}{l}\text { Distrito } \\
\text { XXI, }\end{array}$ & 6,310 & $3.77 \%$ \\
\hline 14 & $\begin{array}{l}\text { José Socorro Ramírez } \\
\text { González }\end{array}$ & México & $\begin{array}{l}\text { Distrito } \\
\text { XXVI }\end{array}$ & 789 & $1.07 \%$ \\
\hline 15 & Joel Hernández Fabian & Michoacán & $\begin{array}{l}\text { Distrito } \\
\text { XXIV }\end{array}$ & 2,076 & $3.85 \%$ \\
\hline 16 & $\begin{array}{l}\text { José Eduardo Santos } \\
\text { González }\end{array}$ & Nuevo León & Distrito VIII & 6,792 & $8.66 \%$ \\
\hline 17 & $\begin{array}{l}\text { Olga Valentina Treviño } \\
\text { Hinojosa }\end{array}$ & Nuevo León & Distrito IX & 6,702 & $9.45 \%$ \\
\hline 18 & $\begin{array}{l}\text { Antonio } \quad \text { González } \\
\text { Quintero }\end{array}$ & Nuevo León & Distrito XIII & 6,814 & $8.66 \%$ \\
\hline 19 & $\begin{array}{l}\text { Martha Magdalena } \\
\text { Montes Salazar }\end{array}$ & Nuevo León & Distrito XIV & 7,785 & $7.96 \%$ \\
\hline 20 & Antonio Román Pereyra & Nuevo León & Distrito XV & 3,886 & $6.95 \%$ \\
\hline 21 & $\begin{array}{l}\text { José Guadalupe } \\
\text { González González }\end{array}$ & Nuevo León & Distrito XVI & 14,594 & $7.50 \%$ \\
\hline 22 & $\begin{array}{l}\text { José Antonio Sánchez } \\
\text { Ortega }\end{array}$ & Nuevo León & $\begin{array}{l}\text { Distrito } \\
\mathrm{XVII}\end{array}$ & 12,655 & $8.93 \%$ \\
\hline 23 & $\begin{array}{l}\text { Tatiana } \quad \text { Clouthier } \\
\text { Carrillo }\end{array}$ & Nuevo León & $\begin{array}{l}\text { Distrito } \\
\mathrm{XVIII}\end{array}$ & 9,488 & $\begin{array}{l}11.97 \\
\%\end{array}$ \\
\hline 24 & Eva Trujillo Ramírez & Nuevo León & Distrito XIX & 8,652 & $9.11 \%$ \\
\hline 25 & $\begin{array}{l}\text { Luis Aurelio Guevara } \\
\text { Garza }\end{array}$ & Nuevo León & Distrito XX & 13,648 & $\begin{array}{l}10.72 \\
\%\end{array}$ \\
\hline 26 & José Luis Rodela Alemán & Nuevo León & Distrito XXI & 869 & $2.29 \%$ \\
\hline
\end{tabular}


Esta obra forma parte del acervo de la Biblioteca Jurídica Virtual del Instituto de Investigaciones Jurídicas de la UNAM www.juridicas.unam.mx

DOI: http://dx.doi.org/10.22201/iij_.24487910e_2015.7.10091

\begin{tabular}{|l|l|l|l|l|l|}
\hline Núm. & $\begin{array}{l}\text { Candidato } \\
\text { independiente }\end{array}$ & Entidad & Distrito & Votación & $\%$ \\
\hline 27 & $\begin{array}{l}\text { Carlos Luis Cabanillas } \\
\text { Herrera }\end{array}$ & Sonora & Distrito XII & 1,365 & 2.76 \\
\hline 28 & $\begin{array}{l}\text { Jorge Alberto Ponce } \\
\text { Salazar }\end{array}$ & Sonora & Distrito XV & 3,225 & 8.66 \\
\hline 29 & Esteban Sánchez Álvarez & Tabasco & Distrito V & 408 & $0.91 \%$ \\
\hline
\end{tabular}

Fuente: http://portales.te.gob.mx/candidaturas-independientes/node/72

El único candidato electo fue José Pedro Kumamoto Aguilar en el Distrito $X$, en Jalisco, con 57,215 votos (37.52\%). Sin embargo, 18 de los 29 candidatos alcanzaron un porcentaje de votación superior al $3 \%$, y en dos casos rebasaron el $10 \%$, teniendo como votación media un porcentaje de 6.71 , por lo que se considera que estos candidatos también resultaron competitivos en algunos distritos locales. Finalmente, respecto a la elección de ayuntamientos, 79 fórmulas obtuvieron su registro en 15 de las 17 entidades federativas. A continuación, se incluye la lista de los candidatos que encabezaron las fórmulas y la votación obtenida:

\section{Cuadro 5}

Cómputos distritales candidatos independientes en los ayuntamientos

\begin{tabular}{|l|l|l|l|l|l|}
\hline Núm. & $\begin{array}{l}\text { Candidato } \\
\text { independiente }\end{array}$ & Entidad & Municipio & Votación & $\begin{array}{l}\text { Porcent } \\
\text { aje }\end{array}$ \\
\hline 1 & $\begin{array}{l}\text { María Herlinda Torrez } \\
\text { Gutiérrez }\end{array}$ & $\begin{array}{l}\text { Baja California } \\
\text { Sur }\end{array}$ & Mulegé & 151 & $0.67 \%$ \\
\hline 2 & $\begin{array}{l}\text { Angélica Ramírez } \\
\text { Gálvez }\end{array}$ & Chiapas Ángel Albino & 379 & $3.03 \%$ \\
\hline 3 & Román García Juárez & Chiapas & Francisco León & 871 & $23.49 \%$ \\
\hline 4 & $\begin{array}{l}\text { Salvador Olvera } \\
\text { Albores }\end{array}$ & Chiapas & Palenque & 780 & $1.72 \%$ \\
\hline 5 & $\begin{array}{l}\text { Juan Manuel Maza } \\
\text { Palacios }\end{array}$ & Chiapas & San Fernando & 665 & $3.68 \%$ \\
\hline
\end{tabular}


Esta obra forma parte del acervo de la Biblioteca Jurídica Virtual del Instituto de Investigaciones Jurídicas de la UNAM www.juridicas.unam. $\mathrm{mx}$

DOl: http://dx.doi.org/10.22201/iij.24487910e_2015.7.10091

\begin{tabular}{|c|c|c|c|c|c|}
\hline Núm. & $\begin{array}{l}\text { Candidato } \\
\text { independiente }\end{array}$ & Entidad & Municipio & Votación & $\begin{array}{l}\text { Porcent } \\
\text { aje }\end{array}$ \\
\hline 6 & $\begin{array}{l}\text { Tomás Abelino } \\
\text { Becerra Gómez }\end{array}$ & Chiapas & Tapachula & 1,594 & $1.62 \%$ \\
\hline 7 & $\begin{array}{l}\text { Felipe de Jesús Juárez } \\
\text { Morales }\end{array}$ & Chiapas & Tecpatán & 2,744 & $24.79 \%$ \\
\hline 8 & $\begin{array}{l}\text { Rafael Jiménez } \\
\text { Arechar }\end{array}$ & Chiapas & $\begin{array}{l}\text { Tuxtla } \\
\text { Gutiérrez }\end{array}$ & 3,799 & $2.23 \%$ \\
\hline 9 & $\begin{array}{l}\text { Agustín Alonso Ramos } \\
\text { Morales }\end{array}$ & Chiapas & $\begin{array}{l}\text { Venustiano } \\
\text { Carranza }\end{array}$ & 491 & $2.02 \%$ \\
\hline 10 & $\begin{array}{l}\text { Alejandro Vinay } \\
\text { Melgar }\end{array}$ & Distrito Federal & Cuajimalpa & 2,431 & $3.26 \%$ \\
\hline 11 & $\begin{array}{l}\text { Nazario Norberto } \\
\text { Sánchez }\end{array}$ & Distrito Federal & $\begin{array}{l}\text { Gustavo A. } \\
\text { Madero }\end{array}$ & 16,656 & $3.81 \%$ \\
\hline 12 & $\begin{array}{l}\text { Rosario Ericka Gómez } \\
\text { Romero }\end{array}$ & Distrito Federal & Milpa Alta & 583 & $1.19 \%$ \\
\hline 13 & Jorge Rivera Olivos & Distrito Federal & Milpa Alta & 474 & $0.96 \%$ \\
\hline 14 & $\begin{array}{l}\text { Juan David Esquivel } \\
\text { Atilano }\end{array}$ & Distrito Federal & Milpa Alta & 170 & $0.34 \%$ \\
\hline 15 & $\begin{array}{l}\text { Oscar Antonio Valdés } \\
\text { Jiménez }\end{array}$ & Distrito Federal & Milpa Alta & 2,874 & $5.87 \%$ \\
\hline 16 & $\begin{array}{l}\text { Arné Sidney Aus Den } \\
\text { Ruthen Haag }\end{array}$ & Distrito Federal & Miguel Hidalgo & 9,657 & $5.59 \%$ \\
\hline 17 & Regina Muñoz García & Guanajuato & Pénjamo & 3,357 & $5.94 \%$ \\
\hline 18 & $\begin{array}{l}\text { José Alberto Méndez } \\
\text { Pérez }\end{array}$ & Guanajuato & Comonfort & 7,873 & $30.03 \%$ \\
\hline 19 & Marcelino Silva Nava & Guerrero & Ahuacuotzingo & 74 & $0.62 \%$ \\
\hline 20 & Ismael Vázquez Mejía & Guerrero & $\begin{array}{l}\text { Cuetzala del } \\
\text { Progreso }\end{array}$ & 664 & $16.32 \%$ \\
\hline
\end{tabular}


Esta obra forma parte del acervo de la Biblioteca Jurídica Virtual del Instituto de Investigaciones Jurídicas de la UNAM www.juridicas.unam.mx

DOl: http://dx.doi.org/10.22201/iij.24487910e_2015.7.10091

\begin{tabular}{|c|c|c|c|c|c|}
\hline Núm. & $\begin{array}{l}\text { Candidato } \\
\text { independiente }\end{array}$ & Entidad & Municipio & Votación & $\begin{array}{l}\text { Porcent } \\
\text { aje }\end{array}$ \\
\hline 21 & Rubén Aranda Álvarez & Guerrero & $\begin{array}{l}\text { Pedro Ascencio } \\
\text { Alquisiras }\end{array}$ & 76 & $2.08 \%$ \\
\hline 22 & $\begin{array}{l}\text { Guillermo López } \\
\text { Cienfuegos } \\
\text { "Lagrimita" }\end{array}$ & Jalisco & Guadalajara & 4,835 & $0.72 \%$ \\
\hline 23 & $\begin{array}{l}\text { José Francisco } \\
\text { Sánchez Peña }\end{array}$ & Jalisco & Puerto Vallarta & 4,213 & $4.62 \%$ \\
\hline 24 & $\begin{array}{l}\text { Jesús Oswaldo Silva } \\
\text { Magaña }\end{array}$ & Jalisco & Tuxpan & 1,407 & $8.68 \%$ \\
\hline 25 & José Zepeda Contreras & Jalisco & Valle de Juárez & 515 & $14.39 \%$ \\
\hline 26 & $\begin{array}{l}\text { Leobardo Javier } \\
\text { Valencia Lozada }\end{array}$ & México & Acolman & 2,644 & $6.50 \%$ \\
\hline 27 & $\begin{array}{ll}\text { Francisco Javier } \\
\text { Santos Arreola }\end{array}$ & México & Cuautitlán & 0 & 0 \\
\hline 28 & $\begin{array}{l}\text { Gustavo Arrieta } \\
\text { Bernal }\end{array}$ & México & Rayón & 251 & $4.13 \%$ \\
\hline 29 & Germán Frías López & México & $\begin{array}{l}\text { San Antonio La } \\
\text { Isla }\end{array}$ & 81 & $0.92 \%$ \\
\hline 30 & Rogelio Morales Brito & México & Tejupilco & 939 & $2.77 \%$ \\
\hline 31 & $\begin{array}{l}\text { Aristóteles Ayala } \\
\text { Rivera }\end{array}$ & México & Texcoco & 3,730 & $3.49 \%$ \\
\hline 32 & $\begin{array}{ll}\text { Vicente } & \text { Espinoza } \\
\text { Hernández } & \end{array}$ & México & Texcoco & 863 & $0.80 \%$ \\
\hline 33 & $\begin{array}{l}\text { José Antonio Medina } \\
\text { Vega }\end{array}$ & México & Villa del Carbón & 2,879 & $14.17 \%$ \\
\hline 34 & $\begin{array}{l}\text { María Guadalupe } \\
\text { Castillo }\end{array}$ & México & Villa Guerrero & 452 & $1.78 \%$ \\
\hline
\end{tabular}


Esta obra forma parte del acervo de la Biblioteca Jurídica Virtual del Instituto de Investigaciones Jurídicas de la UNAM www.juridicas.unam.mx

DOl: http://dx.doi.org/10.22201/iij.24487910e_2015.7.10091

\begin{tabular}{|c|c|c|c|c|c|}
\hline Núm. & $\begin{array}{l}\text { Candidato } \\
\text { independiente }\end{array}$ & Entidad & Municipio & Votación & $\begin{array}{l}\text { Porcent } \\
\text { aje }\end{array}$ \\
\hline 35 & $\begin{array}{l}\text { Miguel Ángel Ortega } \\
\text { López }\end{array}$ & Michoacán & Penjamillo & 331 & $3.84 \%$ \\
\hline 36 & $\begin{array}{l}\text { Cruz Octavio } \\
\text { Rodríguez Castro }\end{array}$ & Michoacán & Purúandiro & 0 & 0 \\
\hline 37 & Jesús Verduzco Duarte & Michoacán & Jacona & 675 & $2.95 \%$ \\
\hline 38 & Marcelo Yepez Salinas & Michoacán & Huaniqueo & 1,340 & $28.87 \%$ \\
\hline 39 & Abel Osorio Soto & Michoacán & Zitácuaro & 1,491 & $2.46 \%$ \\
\hline 40 & $\begin{array}{l}\text { Melitón Naranjo } \\
\text { Rivera }\end{array}$ & Michoacán & $\begin{array}{l}\text { Salvador } \\
\text { Escalante }\end{array}$ & 3,677 & $18.77 \%$ \\
\hline 41 & $\begin{array}{l}\text { Alfonso Jesús } \\
\text { Martínez Alcázar }\end{array}$ & Michoacán & Morelia & 75,380 & $27.56 \%$ \\
\hline 42 & $\begin{array}{l}\text { Ismael Garduño } \\
\text { Ortega }\end{array}$ & Michoacán & Carácuaro & 589 & $10.98 \%$ \\
\hline 43 & $\begin{array}{l}\text { Gilberto Guillén } \\
\text { Villaseñor }\end{array}$ & Michoacán & Acuitzio & 1,527 & $28.70 \%$ \\
\hline 44 & $\begin{array}{l}\text { Fidencio Domínguez } \\
\text { Aguirre }\end{array}$ & Michoacán & Tumbiscatio & 11 & $0.27 \%$ \\
\hline 45 & Martín Torres Causor & Michoacán & $\begin{array}{l}\text { Lázaro } \\
\text { Cárdenas }\end{array}$ & 3,236 & $6.01 \%$ \\
\hline 46 & $\begin{array}{l}\text { Adolfo Navarrete } \\
\text { Torres }\end{array}$ & Morelos & Atlatlahucan & 637 & $6.54 \%$ \\
\hline 47 & $\begin{array}{l}\text { Saul Sotero Medina } \\
\text { Villagómez }\end{array}$ & Morelos & Jojutla & 667 & $2.61 \%$ \\
\hline 48 & $\begin{array}{l}\text { Mahelet Enríquez } \\
\text { Sánchez }\end{array}$ & Morelos & Jonatepec & 1,673 & $20.14 \%$ \\
\hline 49 & $\begin{array}{l}\text { Luis Fernando Marín } \\
\text { Molina }\end{array}$ & Nuevo León & Apodaca & 19,629 & $10.12 \%$ \\
\hline
\end{tabular}


Esta obra forma parte del acervo de la Biblioteca Jurídica Virtual del Instituto de Investigaciones Jurídicas de la UNAM www.juridicas.unam.mx

DOl: http://dx.doi.org/10.22201/iij.24487910e_2015.7.10091

\begin{tabular}{|c|c|c|c|c|c|}
\hline Núm. & $\begin{array}{l}\text { Candidato } \\
\text { independiente }\end{array}$ & Entidad & Municipio & Votación & $\begin{array}{l}\text { Porcent } \\
\text { aje }\end{array}$ \\
\hline 50 & $\begin{array}{l}\text { Miguel Ángel González } \\
\text { Sandoval }\end{array}$ & Nuevo León & El Carmen & 586 & $7.65 \%$ \\
\hline 51 & $\begin{array}{l}\text { César Adrián Valdéz } \\
\text { Martínez }\end{array}$ & Nuevo León & García & 25,557 & $41.74 \%$ \\
\hline 52 & $\begin{array}{l}\text { Lorenia Beatriz } \\
\text { Canavati Von Borstel }\end{array}$ & Nuevo León & $\begin{array}{l}\text { San Pedro } \\
\text { Garza García }\end{array}$ & 11,260 & $17.28 \%$ \\
\hline 53 & $\begin{array}{l}\text { Gabriel Eduardo } \\
\text { Almaguer Segura }\end{array}$ & Nuevo León & Gral. Escobedo & 25,805 & $20.09 \%$ \\
\hline 54 & $\begin{array}{l}\text { Genaro Alanís De la } \\
\text { Fuente }\end{array}$ & Nuevo León & Guadalupe & 21,107 & $6.87 \%$ \\
\hline 55 & $\begin{array}{l}\text { Luis Eduardo Prado } \\
\text { Casanova }\end{array}$ & Nuevo León & Hualahuises & 388 & $9.33 \%$ \\
\hline 56 & $\begin{array}{l}\text { Juan Ignacio Cantú de } \\
\text { Ochoa }\end{array}$ & Nuevo León & Montemorelos & 1,458 & $5.34 \%$ \\
\hline 57 & $\begin{array}{l}\text { Efrén } \quad \text { García } \\
\text { Rodríguez }\end{array}$ & Nuevo León & Santa Catarina & 13,356 & $12.27 \%$ \\
\hline 58 & Alfonso Jiménez Pérez & Nuevo León & Santiago & 1,215 & $5.70 \%$ \\
\hline 59 & $\begin{array}{l}\text { Andrés Sánchez } \\
\text { Sánchez }\end{array}$ & Querétaro & Tolimán & 459 & $3.51 \%$ \\
\hline 60 & $\begin{array}{l}\text { Juan Carlos García } \\
\text { Arellano }\end{array}$ & Querétaro & $\begin{array}{l}\text { Ezequiel } \\
\text { Montes }\end{array}$ & 553 & $2.94 \%$ \\
\hline 61 & $\begin{array}{ll}\text { Israel } & \text { Guerrero } \\
\text { Bocanegra } & \end{array}$ & Querétaro & Tolimán & 1,314 & $10.05 \%$ \\
\hline 62 & $\begin{array}{l}\text { Hugo Amado Muñoz } \\
\text { Flores }\end{array}$ & Querétaro & $\begin{array}{l}\text { Cadereyta de } \\
\text { Montes }\end{array}$ & 6,659 & $22.91 \%$ \\
\hline 63 & $\begin{array}{l}\text { Hipólito Rigoberto } \\
\text { Pérez Montes }\end{array}$ & Querétaro & $\begin{array}{l}\text { Ezequiel } \\
\text { Montes }\end{array}$ & 2,800 & $14.88 \%$ \\
\hline
\end{tabular}


Esta obra forma parte del acervo de la Biblioteca Jurídica Virtual del Instituto de Investigaciones Jurídicas de la UNAM www.juridicas.unam.mx

DOl: http://dx.doi.org/10.22201/iij.24487910e_2015.7.10091

\begin{tabular}{|c|c|c|c|c|c|}
\hline Núm. & $\begin{array}{l}\text { Candidato } \\
\text { independiente }\end{array}$ & Entidad & Municipio & Votación & $\begin{array}{l}\text { Porcent } \\
\text { aje }\end{array}$ \\
\hline 64 & $\begin{array}{l}\text { Federico Montero } \\
\text { Castillo }\end{array}$ & Querétaro & Corregidora & 2,722 & $4.14 \%$ \\
\hline 65 & $\begin{array}{l}\text { José Carlos Laborde } \\
\text { Vega }\end{array}$ & Querétaro & El Marqués & 567 & $1.01 \%$ \\
\hline 66 & $\begin{array}{ll}\text { Faustino } & \text { Fuentes } \\
\text { Gutiérrez } & \end{array}$ & San Luis Potosí & Ciudad Maíz & 257 & $1.82 \%$ \\
\hline 67 & $\begin{array}{l}\text { María del Carmen } \\
\text { Flores González }\end{array}$ & San Luis Potosí & Ébano & 120 & $0.65 \%$ \\
\hline 68 & Joel Fuentes Cruz & Sonora & Empalme & 1,242 & $5.93 \%$ \\
\hline 69 & $\begin{array}{l}\text { Reyes Fredy Molina } \\
\text { Martínez }\end{array}$ & Sonora & Fronteras & 449 & $10.76 \%$ \\
\hline 70 & $\begin{array}{l}\text { Crispín Montenegro } \\
\text { Romero }\end{array}$ & Sonora & $\begin{array}{l}\text { San Miguel de } \\
\text { Horcasitas }\end{array}$ & 12 & $0.40 \%$ \\
\hline 71 & $\begin{array}{l}\text { Carlos Arturo Vara } \\
\text { López }\end{array}$ & Sonora & $\begin{array}{l}\text { San Ignacio Río } \\
\text { Muerto }\end{array}$ & 1,863 & $27.10 \%$ \\
\hline 72 & Oscar Sánchez Peralta & Tabasco & Cárdenas & 4,107 & $4.45 \%$ \\
\hline 73 & $\begin{array}{l}\text { Felipe Sánchez } \\
\text { Alejandro }\end{array}$ & Tabasco & Centla & 338 & $0.75 \%$ \\
\hline 74 & $\begin{array}{l}\text { Pedro Antonio } \\
\text { Contreras López }\end{array}$ & Tabasco & Centro & 2,056 & $0.96 \%$ \\
\hline 75 & $\begin{array}{l}\text { Francisco Córdova } \\
\text { Broca }\end{array}$ & Tabasco & Comalcalco & 930 & $1.03 \%$ \\
\hline 76 & Limber Peláez Zurita & Tabasco & Macuspana & 4,723 & $8.42 \%$ \\
\hline 77 & $\begin{array}{l}\text { Luis Arturo de la } \\
\text { Fuente Sánchez }\end{array}$ & Tabasco & Paraíso & 741 & $18.02 \%$ \\
\hline 78 & $\begin{array}{l}\text { Gabriel Arturo May } \\
\text { Pech }\end{array}$ & Yucatán & Yaxkukul & 43 & $2.12 \%$ \\
\hline
\end{tabular}


Esta obra forma parte del acervo de la Biblioteca Jurídica Virtual del Instituto de Investigaciones Jurídicas de la UNAM www.juridicas.unam.mx

DOI: http://dx.doi.org/10.22201/iij_.24487910e.2015.7.10091

\begin{tabular}{|l|l|l|l|l|l|}
\hline Núm. & $\begin{array}{l}\text { Candidato } \\
\text { independiente }\end{array}$ & Entidad & Municipio & Votación & $\begin{array}{l}\text { Porcent } \\
\text { aje }\end{array}$ \\
\hline 79 & José Raúl Canul Dzul & Yucatán & Acanceh & 497 & $6.37 \%$ \\
\hline
\end{tabular}

Fuente: http://portales.te.gob.mx/candidaturas-independientes/node/72

De los 79 candidatos a presidente municipal sólo 3 resultaron electos: Alfonso Jesús Martínez Alcázar en el municipio de Comonfort en Guanajuato; José Alberto Méndez Pérez en el municipio de Morelia, en Michoacán, y César Adrián Valdez Martínez en el municipio de García en Nuevo León, teniendo como porcentaje medio de votación un $3.03 \%$, el más bajo de todos los grupos de candidatos independientes aquí revisados.

En total fueron 133 ciudadanos que lograron el registro como candidatos independientes en el proceso electoral 2014-2015, de los cuales sólo resultaron electos 6 (4.5\%): 1 diputado federal, 1 gobernador, 1 diputado local y 3 presidentes municipales.

En la siguiente gráfica puede observarse el número de candidatos independientes registrados por entidad federativa. Es de resaltar que sólo en Colima no se registró candidato independiente alguno, lo que indica que la figura fue utilizada de manera casi generalizada en las entidades donde hubieron procesos electorales. A futuro, resultará necesario analizar el caso de Nuevo León no sólo por la victoria del candidato a gobernador sino porque en esa entidad obtuvieron su registro 22 candidatos, el doble de las entidades que se ubicaron en segundo lugar en cuanto al número de candidatos registrados.

\section{Figura 2}

Entidades federativas en orden decreciente por número de candidatos independientes

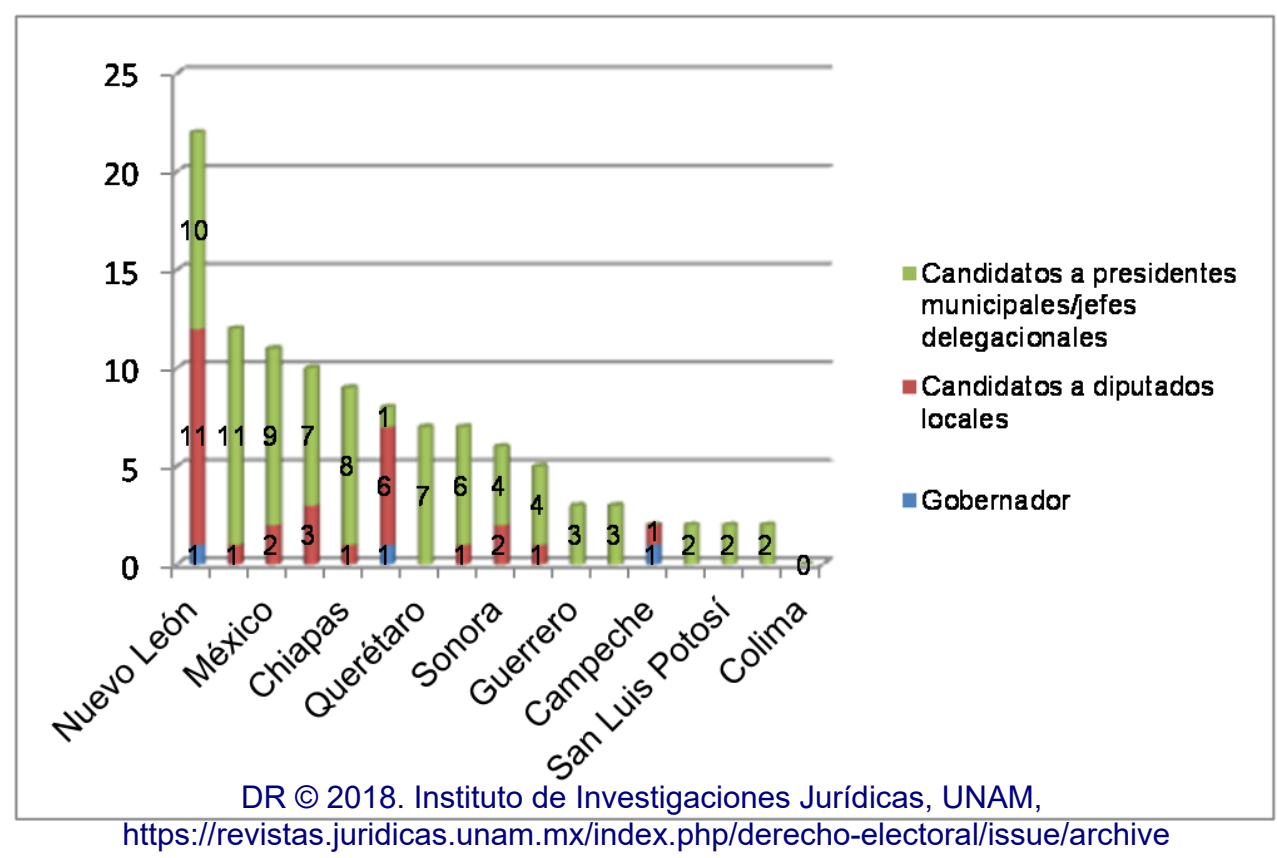


Esta obra forma parte del acervo de la Biblioteca Jurídica Virtual del Instituto de Investigaciones Jurídicas de la UNAM

Fuente: elaboración propia con datos de http://portales.te.gob.mx/candidaturasindependientes/

\section{EFECTIVIDAD DE LAS CANDIDATURAS INDEPENDIENTES FRENTE A LOS OBJETIVOS DE LA REFORMA}

El proceso electoral 2014-2015 no sólo ha sido importante por la concurrencia de los 17 procesos locales ordinarios con la elección federal, sino también porque es el primer proceso electoral que se celebró después de la promulgación de la reforma constitucional en material político electoral de 2014.

Los fines primordiales de las iniciativas en materia electoral eran reformar la Constitución para fortalecer el Estado democrático mediante la participación ciudadana, la legitimación de las instituciones democráticas, la equidad en las contiendas y la resolución de los problemas de carácter promocional que surgieron durante la elección federal de 2012, y las elecciones locales de 2013. De entre los múltiples cambios introducidos con motivo de la reforma político-electoral de 2014, se encuentra la inclusión de las bases para las candidaturas independientes. Esta inclusión tiene como antecedente, la reforma constitucional en materia de derechos humanos de 2011, a partir de la cual las autoridades deben basarse en la progresividad y el respeto de éstos, regir sus actos por el principio pro homine y garantizar que los individuos gocen de tales derechos de la forma más amplia de conformidad con la Constitución y los tratados internacionales en materia de derechos humanos.

Así, la comunidad internacional reconoció que los derechos políticos son parte de los derechos humanos, y por lo tanto tienen la función de garantizar al ciudadano de un Estado la capacidad de actuar, decidir, participar e influir en la administración de los poderes públicos, a través de los cuales se organiza. ${ }^{11}$ Adicionalmente las personas que buscaban un avance en la democracia del país encontraron en las candidaturas independientes una utilidad indiscutible para ello, fundamentalmente para consolidar y mejorar la calidad democrática, ya que aumenta la participación de los ciudadanos, se

\footnotetext{
${ }^{11}$ Carrasco Daza, Constancio, “¿Un nuevo régimen de las candidaturas independientes a partir del reconocimiento constitucional de los derechos humanos?" en Cienfuegos Salgado, David y Froto Madariaga, Germán (coords.), Los derechos humanos en el momento actual, México, Comisión de Derechos Humanos del Estado de Coahuila, Poder Judicial del Estado de Coahuila, Universidad Autónoma de Coahuila, Editorial Laguna, 2012, p.115.
} 
Esta obra forma parte del acervo de la Biblioteca Jurídica Virtual del Instituto de Investigaciones Jurídicas de la UNAM www.juridicas.unam.mx

reconoce el derecho que tienen las personas de participar en la política del país, sin la obligación de afiliarse a un partido político y se incrementan las opciones para el electorado. $^{12}$

Visto el resultado obtenido en el proceso electoral (2014-2015) se afirma que éste se debe a la inclusión de las candidaturas independientes, las cuales a pesar de las deficiencias legislativas observadas por varios analistas y críticos nacionales, dieron pie a una nueva alternativa política en nuestro sistema jurídico, la cual deberá reglamentarse de manera adecuada en el futuro, para formar parte de manera definitiva en nuestro sistema jurídico político-electoral.

Resulta relevante mencionar específicamente el triunfo obtenido en la elección de gobernador del estado de Nuevo León en el cual resultó ganador, Jaime Humberto Rodríguez Calderón, quien había pertenecido a las filas del Partido Revolucionario Institucional y al no tener asignación por ningún partido acudió como independiente y ganó la elección con una diferencia sobresaliente respecto de sus contendientes, todos ellos partidos políticos. Es conocida la importancia económica y social de la ciudadanía del estado de Nuevo León que se vio identificada con esta candidatura independiente y no con algún partido político.

\section{FUENTES CONSULTADAS}

\section{Bibliografía}

Astudillo, César, "La postulación de candidaturas partidistas e independientes en América Latina en el contexto de los derechos de participación política", en Freidenberg, Flavia y Muñoz Pogossian, Betilde (eds.), Las reformas a las organizaciones de partidos en América Latina, Lima, Pontificia Universidad Católica, Organización de los Estados Americanos, UNAM, Instituto de Investigaciones Jurídicas, Sociedad Argentina de Análisis Político, 2015.

Becerra, Pablo, “Las candidaturas independientes en México. Una vía para ampliar la participación ciudadana", en GILAS, Karolina y MEDINA TORRES, Eduardo (eds.), Candidaturas independientes: desafíos y propuestas, México, TEPJF, Instituto Electoral de Morelos, Tirant lo Blanch, 2014.

\footnotetext{
12 Ibidem, p. 123; Hernández Olmos, Mariana, La importancia de las candidaturas independientes, núm. 12, Cuadernos de Divulgación de Justicia Electoral, México, 2012, pp. 49 y 368.
} 
Esta obra forma parte del acervo de la Biblioteca Jurídica Virtual del Instituto de Investigaciones Jurídicas de la UNAM www.juridicas.unam.mx

BhatTACHARYA, Kaushik, "Independent Candidates in a Parliamentary Election in India: A Poisson Regression Model", Working Paper Series: 2009/10-034, Prabandh Nagar, Indian Institute of Management Lucknow, 2010.

Bolleyer, Nicole y WeEKS, Liam, "The Puzzle of Non-Party Actors in Party Democracy: Independents in Ireland", Comparative European Politics, vol. 7 (3), 2007.

BRANCATI, Dawn, "Winning Alone: The Electoral Fate of Independent Candidates Worldwide", Journal of Politics, vol. 70 (3), 2008.

CarRasco Cruz, Arturo, "Las candidaturas independientes en México", Huerta Ladrón de Guevara, 2014.

CARRASco DazA, Constancio, “¿Un nuevo régimen de las candidaturas independientes a partir del reconocimiento constitucional de los derechos humanos?", en CIENFUEGOS SAlgado, David y Froto MAdARIAGA, Germán, (coords.), Los derechos humanos en el momento actual, México, Comisión de Derechos Humanos del Estado de Coahuila, Poder Judicial del Estado de Coahuila, Universidad Autónoma de Coahuila, Editorial Laguna, 2012.

DOŠEK, Tomáš, "La presencia y el éxito de los independientes en las elecciones subnacionales en Chile (2004-2012)", XI Congreso Chileno de Ciencia Política (ACCP), Política, diversidad y conflicto, nuevos debates a 30 años de la ACCP, Santiago de Chile, 15 al 17 de octubre de 2014.

HALE, Henry, Why not Parties in Russia?, Cambridge, Cambridge University Press, 2007.

HERNÁNDEZ Olmos, Mariana, La importancia de las candidaturas independientes, núm. 12, Cuadernos de Divulgación de Justicia Electoral, México, 2012.

ISHIYAMA, John, BATTA, Anna et al., "Political Parties, Independents and the Electoral Market in Sub-Saharan Africa", Party Politics, vol. 19 (5), 2011.

MOSER, Robert, "Independents and Party Formation: Elite Partisanship as an Intervening Variable in Russian Politics", Comparative Politics, vol. 31 (2), 1999.

RODRIGUES, Mark y BRENTON, Scott, “The Age of Independence? Independents in Australian Parliaments", Australasian Parliamentary Review, vol. 25 (1), 2010.

WeEks, Liam, “We don't like (to) party. A typology of Independents in Irish Political Life, 1922-2007", Irish Political Studies, vol. 24 (1), 2009. 
Esta obra forma parte del acervo de la Biblioteca Jurídica Virtual del Instituto de Investigaciones Jurídicas de la UNAM www.juridicas.unam.mx

\section{Normatividad}

Constitución Política de los Estados Unidos Mexicanos. Actualizada con la última reforma del 7 de junio de 2014, http://Www.diputados.gob.mx/LeyesBiblio/pdf/1_07ju/14.pdf (consultada el 4 de agosto de 2015).

Diario Oficial de la Federación del 9 de agosto del 2012, Decreto por el que se reforman, adicionan y derogan diversas disposiciones de la CPEUM, en materia política-electoral, México, Secretaría de Gobernación.

Ley Electoral del Estado de Baja California Sur, http://Www.ieebcs.org.mx/documentos/legis/acion/IEEBCS_LEG26.pdf (consultada el 4 de agosto de 2015).

Ley Electoral para el Estado de Nuevo León, http://Www.cee.nl.org.mx/legis/acion/documentos/leyes/LEY\%20ELECTORAL\%2ONUEV O\%20LEON\%20CEE\%20EDICION\%20FINAL.pdf(consultada el 4 de agosto de 2015).

Ley Electoral del Estado de Querétaro. Actualizada con la última reforma publicada el 29 de junio de 2014, http://Www.ieeq.mx/ieq/contenido/marco/archivos/leyes/LEEQ.pdf (consultada el 4 de agosto de 2015).

Ley General de Instituciones y Procedimientos Electorales. Actualizada con la última reforma publicada el 23 de mayo de 2014, http://Www.diputados.gob.mx/LeyesBiblio/pdf/LGIPE_100914.pdf (consultada el 4 de agosto de 2015).

Ley de Instituciones y Procedimientos Electorales del Estado de Campeche, http://Www.ieec.org.mx/LeyInstProcElectorales.pdf (consultada el 4 de agosto de 2015).

Acuerdos y resoluciones

Acciones de inconstitucionalidad SCJN: acción de inconstitucionalidad 22/2014 y sus acumuladas 26/2014, 28/2014 y 30/2014.

Acuerdo INE: INE/ACRT/17/2015.

Informe sobre el registro de candidaturas independientes en el proceso electoral federal 2014-2015 y las acciones realizadas para garantizar su financiamiento público y el acceso a la franquicia postal, en cumplimiento al punto quinto del acuerdo 
Esta obra forma parte del acervo de la Biblioteca Jurídica Virtual del Instituto de Investigaciones Jurídicas de la UNAM www.juridicas.unam. $m x$

\section{INE/CG88/2015.}

Sentencias relevantes TEPJF: SUP-JDC-37/2001, SUP-JDC-86/2007, SUP-JDC-713/2014, SMJDC-429/2015 y SUP-JDC-838/2015. 